\title{
A Construção de uma Escala de Estratégias de Aprendizagem para Alunos do Ensino Fundamental ${ }^{1}$
}

\author{
Evely Boruchovitch ${ }^{2}$ \\ Universidade Estadual de Campinas \\ Acácia Aparecida Angeli dos Santos \\ Universidade São Francisco \\ Elis Regina da Costa \\ Faculdade de Ciências Sociais e Agrárias de Itapeva \\ Edna Rosa Correia Neves \\ Miriam Cruvinel \\ Universidade Estadual de Campinas \\ Ricardo Primi \\ Universidade São Francisco \\ Sueli Edi Rufini Guimarães \\ Universidade Estadual de Londrina
}

\begin{abstract}
RESUMO - Embora as estratégias de aprendizagem sejam importantes para o processo de escolarização, existe uma carência de instrumentos nacionais que possibilitem um conhecimento do repertório referente a elas em estudantes brasileiros. Assim sendo, os objetivos deste trabalho são: descrever os passos relativos à construção de uma escala para avaliar as estratégias de aprendizagem de alunos do ensino fundamental e apresentar o estudo preliminar de suas propriedades psicométricas. Discute-se a utilidade desse instrumento para diagnóstico, intervenção e prevenção em psicologia escolar e educacional.
\end{abstract}

Palavras-chave: psicometria; construção de teste; ensino fundamental.

\section{The Construction of a Learning Strategy Scale for Basic Education Students}

\begin{abstract}
Although learning strategies are important tools for schooling process, there is a lack of national instruments to evaluate their knowledge by brazilian students. Therefore, the objectives of this paper are to describe the steps necessary for the construction of a scale to evaluate the learning strategies for basic education students and to present the preliminary study of its psychometric properties. It is also discussed the utility of this instrument for diagnosis, intervention and prevention in school and educational psychology.
\end{abstract}

Key words: psychometric; test construction; basic education.

As pesquisas brasileiras sobre estratégias de aprendizagem, embora tenham aumentado recentemente, ainda são escassas, em todas as etapas da escolarização. Alguns estudos nacionais vêm sendo realizados no sentido de conhecer o que alunos fazem espontaneamente para aprender e estudar em diversas situações específicas de aprendizagem e estudo (Boruchovitch, 1995, 2001; Chakur, 1998; Costa, 2000; Costa \& Boruchovitch, 2004; Cruvinel \& Boruchovitch, 2004, Escolano \& Linhares, 1998; Gombi, 1999; Gomes \& Boruchovitch, 2005; Jalles, 1997; Joly, Cantalice \& Vendramini, 2004; Joly \& Paula, 2005; Lopes, 1997; Machado, 2005; Molina, 1983, 1984, Schlieper, 2001).

Estratégias de aprendizagem são seqüências de procedi-

1 Os autores agradecem o apoio financeiro do CNPq

2 Endereço: Rua Coronel Quirino 910, apto. 24. Cambuí, Campinas, SP, Brasil,13.025-001. E-mail:evely@unicamp.br mentos ou atividades que se escolhem com o propósito de facilitar a aquisição, o armazenamento e a utilização da informação (Pozo, 1996). Têm um caráter consciente e intencional e em nível mais específico, podem ser consideradas como qualquer procedimento adotado para a realização de uma tarefa (Da Silva \& De Sá, 1997). Alguns teóricos distinguem as estratégias cognitivas das metacognitivas (Garner \& Alexander, 1989). Para Dembo (1994), enquanto as estratégias cognitivas referem-se a comportamentos e pensamentos que influenciam o processo de aprendizagem de maneira que a informação possa ser armazenada mais eficientemente, as estratégias metacognitivas são procedimentos que o indivíduo usa para planejar, monitorar e regular o seu próprio pensamento (Boruchovitch, 1993; 1999).

Da Silva e De Sá (1997) apontam que a instrução em estratégias de aprendizagem possibilita aos estudantes ultrapassar dificuldades pessoais e ambientais de forma a obter um maior sucesso escolar. Estratégias de aprendizagem podem 
ser ensinadas para alunos de baixo rendimento escolar. É possível ensinar a todos os alunos a expandir notas de aulas, a sublinhar pontos importantes de um texto, a monitorar a compreensão da leitura, usar estratégias de memorização, fazer resumos, entre outras.

Resultados de pesquisas revelam que a intervenção para o desenvolvimento de estratégias de aprendizagem tem sido bem sucedida, de modo geral, pois é capaz de produzir, tanto uma melhora imediata em seu uso, quanto no rendimento escolar geral dos alunos (Garner, Hare, Alexander, Haynes \& Winograd, 1984; Pressley \& Levin, 1983; Weinstein \& Mayer, 1985). Ressaltam os teóricos nessa área que apenas conhecer as estratégias não é suficiente para melhorar o rendimento escolar dos estudantes. Faz-se necessário que os alunos compreendam como e quando usá-las.

Se por um lado, o ensino de estratégias de aprendizagem é capaz de melhorar consideravelmente o desempenho dos alunos, por outro lado, não há dúvidas de que a intervenção em estratégias de aprendizagem somente será eficaz para desenvolver a capacidade do aluno para aprender a aprender, se ao ensino de estratégias forem associadas estratégias de apoio afetivo, destinadas a modificar variáveis psicológicas, tais como, ansiedade, auto-eficácia, autoconceito, atribuição de causalidade, muitas vezes incompatíveis ao uso apropriado das estratégias. Deste modo, o ensino de estratégias de aprendizagem pode ser otimizado (Hattie, Biggs \& Purdie, 1996).

Entre os instrumentos disponíveis para mensurar estratégias de aprendizagem na literatura internacional, podem-se destacar como principais, o Learning and Study Strategies Inventory (LASSI) desenvolvido por Weinstein e Palmer (1987; 1990), o Motivated Strategies for Learning Questionnaire (MSLQ) de Pintrich e Groot (1989) e a Self-Regulated Learning Interview Schedule de Zimmerman e Martinez-Pons (1986; Zimmerman, 1986; Zimmerman, 1998).

No Brasil, as primeiras investigações, visando conhecer as estratégias de aprendizagem de alunos do ensino fundamental, foram baseadas em instrumentos traduzidos e adaptados da literatura estrangeira (Boruchovitch, 1995, 2001; Costa, 2000, Costa \& Boruchovitch, 2004; Schlieper, 2001). Tendo em vista a reconhecida importância das estratégias de aprendizagem em todos os níveis de escolarização e a ausência de instrumentos nacionais que possibilitem um conhecimento mais rápido do repertório de estratégias de aprendizagem, desenvolveu-se, inicialmente, uma escala para alunos universitários (Santos \& Boruchovitch, 2001; Santos, Boruchovitch, Primi, Bueno \& Zenorini, 2004) e, também, uma escala infantil (Boruchovitch \& Santos, 2001).

Para a construção das duas escalas, levou-se em conta, criteriosamente, tanto os instrumentos internacionais disponíveis na literatura da área, quanto os resultados provenientes de pesquisas internacionais e nacionais sobre o tema. Estratégias de memorização, de elaboração, de administração de tempo, de estruturação do ambiente, de auto-avaliação, específicas de compreensão da leitura, de apoio afetivo têm sido as principais atividades mencionadas por pesquisadores como fazendo parte do repertório de estratégias de alunos de diversas faixas etárias e diferentes nacionalidades. Tendo em vista sua relevância para a representatividade do construto, essas atividades integram o conteúdo dos itens das escalas existentes (Boruchovitch, 1995; 2001; Chakur, 1998; Costa, 2000; Costa \& Boruchovitch, 2004; Escolano \& Linhares, 1998; Gombi, 1999; Jalles, 1997; Lopes, 1997; Molina, 1983, 1984, Purdie \& Hattie, 1996; Schlieper, 2001).

Considerando-se a importância de se construir instrumentos válidos e fidedignos, que possam estar disponíveis para diagnóstico, intervenção e prevenção em psicologia escolar (Noronha, 2002; Noronha, Oliveira \& Beraldo, 2003, entre outros), os objetivos desse trabalho são descrever os passos relativos à construção de uma escala para avaliar o repertório de estratégias de aprendizagem de alunos do ensino fundamental e apresentar a análise preliminar de suas propriedades psicométricas, o que ocorreu em dois estudos descritos a seguir.

\section{Estudo 1}

\section{Método}

\section{Participantes}

Fizeram parte do estudo 305 alunos de duas escolas do ensino fundamental de Campinas, divididos em dois grupos. Ambas as escolas atendiam alunos provenientes de classe sociais desfavorecidas. O primeiro grupo foi composto de 155 alunos de $2^{\mathrm{a}}$ série $(24,5 \%), 4^{\mathrm{a}}$ série $(25,8 \%), 6^{\mathrm{a}}$ série $(25,8 \%)$ e $8^{\mathrm{a}}$ série $(23,9 \%)$, de ambos os sexos, sendo $51,6 \%$ do sexo masculino e $48,4 \%$ do sexo feminino. Dos participantes desse grupo, 51,6\% nunca havia repetido uma série escolar, enquanto que $43,9 \%$ tinha tido pelo menos uma reprovação. A faixa etária variou de 7 a 18 anos, sendo que 39,4\% dos alunos tinham de 7 a 10 anos, 42,6 \%, entre 11 e 14 anos, e $18,1 \%$, entre 15 a 18 anos. A média da idade dos alunos foi 11,64 e o desvio padrão de 2,98.

O segundo grupo foi composto de 150 estudantes de $3^{\text {a }}$ série $(33,3 \%), 5^{\mathrm{a}}$ série $(33,3 \%)$ e $7^{\mathrm{a}}$ série $(33,3 \%)$ do ensino fundamental, de ambos os sexos, sendo $51,3 \%$ do gênero feminino e $48,7 \%$ do gênero masculino. A faixa etária variou de 9 a 15 anos, sendo que $31,3 \%$ dos estudantes tinham 9 anos, 63,3\% entre 10 a 12 anos e 5,4\%, entre 13 a 15 anos. A média de idade dos participantes foi de 11,32 e o desvio padrão foi de 1,69. A maioria dos participantes desse grupo não havia repetido nenhuma série escolar (76,0\%). Cabe esclarecer que a faixa etária dos alunos do primeiro grupo foi mais ampla (7 a 18 anos) do que a dos estudantes do segundo grupo (9 a 15 anos) porque a escola do primeiro grupo não havia ainda adotado o regime de progressão continuada (LDB - Lei 9394/96, artigo 32, parágrafo segundo).

\section{Instrumento}

Os dados relativos às estratégias de aprendizagem dos participantes foram coletados por meio de uma entrevista estruturada, Self-Regulated Learning Structured Interview 
(Zimmerman \& Martinez-Pons, 1986), traduzida e adaptada por Boruchovitch, (1995). Como esse material foi originalmente escrito em inglês, a autora efetuou sua tradução do inglês para o português num primeiro momento, e, em seguida, solicitou que um outro pesquisador brasileiro que morou num país de língua inglesa, fizesse a versão dos instrumentos para o inglês (back translation). Esse cuidado possibilitou uma adaptação confiável do instrumento, tal como originalmente proposto.

A entrevista era composta de 17 questões abertas, algumas contendo itens fechados do tipo sim e não. As questões dessa entrevista eram baseadas em situações concretas relativas ao ensino e a aprendizagem dos participantes, que, mais precisamente, mapeavam as seguintes situações: a) aprendizagem em sala de aula, b) estudo em casa, c) realização de tarefas escolares em casa e de preparação para provas. Como exemplo, pode se citar a seguinte questão: A maioria dos professores costuma dar testes no final do semestre. Esses testes determinam a nota dos alunos. Você tem alguma maneira que possa lhe ajudar a se preparar para uma prova de português?

\section{Procedimento}

Inicialmente foi realizado um contato com diretores de duas escolas de Campinas, e estabelecido o cronograma de atividades de comum acordo. Foram observados os princípios e procedimentos éticos estabelecidos pelo Conselho Nacional de Saúde (CNS, 1996), tais como o respeito à liberdade e privacidade dos participantes, a não interferência nas rotinas da escola, assim como o caráter confidencial dos dados.

Os aplicadores, especialmente treinados para a tarefa, realizavam a entrevista com cada estudante em uma única sessão com duração de 45 a 60 minutos. Era pedido para o participante que explicasse melhor sempre que a resposta dada fosse ambígua ou vaga. Se o participante desse uma resposta clara, perguntava-se a ele, se costuma fazer mais alguma coisa além das que havia citado, seguindo assim, o procedimento recomendado pelos autores da entrevista original.

É importante ressaltar que era permitido que os participantes mencionassem mais de uma estratégia de aprendizagem para cada situação proposta, se assim o desejassem. O procedimento de coleta de dados e a entrevista foram prétestados em estudo piloto realizado com 60 alunos de $2^{\mathrm{a}}$ a $8^{\mathrm{a}}$ série do ensino fundamental que não fizeram parte da amostra.

\section{Resultados e Discussão}

As respostas dos alunos do primeiro grupo às questões abertas foram examinadas, mediante os procedimentos de análise de conteúdo (Bardin, 1977/1991; Berelson, 1952). Para aumentar a confiabilidade da análise de conteúdo, foi desenvolvido um sistema de categorização de respostas para cada questão. Assim, cada categoria foi definida operacionalmente e um conjunto de regras para a classificação de uma resposta numa dada categoria foi cuidadosamente desenvolvido por Boruchovitch e Costa (Costa, 2000). As respostas dos estudantes foram analisadas à luz do sistema de categorização proposto. Durante a análise dos dados, os pesquisadores não tiveram acesso a informações relativas aos dados demográficos da amostra. Análises mais detalhadas a respeito das relações entre as respostas dos participantes e as variáveis idade, série escolar, gênero e repetência foram também realizadas e podem ser encontradas em Costa (2000), Costa e Boruchovitch (2004) e Schlieper (2001).

A consistência do processo de categorização foi avaliada mediante a utilização de juízes independentes que analisaram $20 \%$ das respostas dos participantes selecionadas aleatoriamente, utilizando o conjunto de regras mencionado. Os juízes também não foram informados sobre os dados relativos à reprovação escolar dos participantes durante o processo de categorização, ou seja, trabalharam às cegas para que essa informação não contaminasse suas análises. A porcentagem de correspondência alcançada entre os juízes e os pesquisadores foi de $90 \%$.O sistema de categorização desenvolvido foi, posteriormente, aplicado às respostas dos participantes do segundo grupo, tendo-se revelado adequado para análise das respostas obtidas na ocasião. Foi novamente realizada uma análise da consistência do processo de categorização das respostas de forma idêntica à primeira, tendo se encontrado o mesmo percentual de concordância entre juízes.

Embora a utilização de questões abertas sempre exija o desenvolvimento de uma análise de conteúdo sistemática e criteriosa, elas permitem um melhor mapeamento das categorias de respostas existentes para uma dada questão. Assim, podem se constituir numa primeira etapa para a construção de instrumentos, possibilitando coletas de dados de forma mais rápida, objetiva e precisa sobre o construto de interesse. Pesquisadores defendem a utilidade das questões abertas, sugerindo que as mesmas funcionem como um estudo piloto, antecedendo a construção de medidas mais objetivas, adequadas e sensíveis à realidade a ser investigada (Boruchovitch \& Schall, 1999; Günther \& Lopes, 1990; Isaac \& Michael, 1982).

Baseada nas informações obtidas em estudos anteriores com questões abertas e na literatura da área, a primeira versão da escala de estratégias de aprendizagem para alunos do ensino fundamental (EAEF) (Boruchovitch \& Santos, 2001) foi construída com o objetivo de avaliar o repertório de estratégias de aprendizagem de alunos, bem como a freqüência com que os estudantes as utilizam. Os autores consideraram que essa etapa poderia ser de grande valia para os passos seguintes da construção do instrumento pretendido.

Vale destacar que antes de dar início ao segundo estudo, os itens elaborados com base nos dados das entrevistas e na literatura da área foram pré-testados em 34 alunos, 30 de $4^{\text {a }}$ série, 2 de $3^{\text {a }}$ série, e 1 de $5^{\text {a }}$ série do ensino fundamental de duas escolas públicas de diferentes cidades, respectivamente por Cruvinel (2003) e Gomes (2002), tendo sido refinados, 
em seguida, no que diz respeito a pequenos problemas de linguagem que foram detectados e implicaram na reformulação da redação de alguns deles. Como exemplo de uma modificação decorrente dessa primeira aplicação, pode-se citar que a palavra "sublinhar" foi substituída pela frase "passar uma linha embaixo das partes importantes do texto".

A escala de estratégias de aprendizagem (EAEF) foi inicialmente construída com 40 itens, propostos em forma de escala likert e uma questão aberta, visando investigar se o aluno usa mais alguma estratégia não contemplada nos itens apresentados. A idéia inicial era de se explorar a funcionalidade, adequação e potencialidades de um maior número de itens, com o propósito de se chegar, num segundo momento, a um instrumento menor e de aplicação menos cansativa para as crianças.

Assim sendo, os itens 1, 2, 5, 7, 12, 13, 15, 16, 33 e 36 englobavam as principais estratégias cognitivas de aprendizagem (ensaio, elaboração e organização). Já os demais itens referiam-se a estratégias metacognitivas como planejamento, monitoramento, controle e regulação da aprendizagem. Após a elaboração das frases, foi feita uma avaliação do conteúdo e uma classificação de cada uma, considerando-se o sistema proposto por Mckeachie, Pintrich, Lin, Smith e Sharma (citados por Dembo, 1994). Com isso, confirmou-se que de fato os itens eram representativos dos principais tipos de estratégias cognitivas e metacognitivas a que se propunham, tanto para tarefas simples quanto para as mais complexas. Foi elaborado também um conjunto de instruções que acompanham a escala, a ser lido pelos aplicadores de forma a garantir a homogeneidade da aplicação do instrumento.

\section{Estudo 2}

\section{Método}

\section{Participantes}

A amostra foi composta de 433 estudantes de $3^{\text {a }}$ a $8^{\text {a }}$ série do ensino fundamental de $3^{\text {a }}$ a $8^{\text {a }}$ série, de ambos os sexos, de nível sócio-econômico médio e baixo, provenientes de escolas públicas do ensino fundamental de Campinas e de Catalão - Goías, sendo 265 alunos de Campinas e 168 de Catalão. A faixa etária variou de 8 a 18 anos. A média de idade foi de 11,65 e o desvio padrão de 2,12 , sendo $32 \%$ dos participantes entre 8 a 10 anos, $47 \%$ entre 11 a 13 anos, $20 \%$ entre 14 e 16 anos e $1 \%$ entre 17 e 18 anos. No que diz respeito à série escolar, $16 \%$ dos alunos encontravam-se na $3^{\mathrm{a}}$ série, $15 \%$ na $4^{\mathrm{a}}$ série, $21 \%$ na $5^{\mathrm{a}}$ série, $15 \%$ na $6^{\mathrm{a}}$ série, $15 \%$ na $7^{\text {a }}$ série e $15 \%$ na $8^{\text {a }}$ série Em relação ao gênero, 56 $\%$ dos participantes eram do sexo masculino e $44 \%$ do sexo feminino.

\section{Instrumento}

Como descrito anteriormente, a escala consta de 40 itens propostos em forma de escala likert e uma questão aberta, visando investigar se o aluno usa mais alguma estratégia não contemplada nos itens apresentados, com três alternativas de respostas para cada item, a saber: sempre, algumas vezes e nunca. Para a resposta "sempre" atribuiuse 3 pontos, para "algumas vezes" 2 pontos e para "nunca" 1 ponto, com exceção das questões $28,30,31,32,35,37$, 38,39 e 40 , cuja pontuação na hora da análise teve seu valor invertido. O valor máximo de pontos obtidos na escala é de 120 pontos e o valor mínimo é de 40 pontos.

\section{Procedimentos}

Seguindo os mesmos procedimentos realizados no Estudo 1 , os pesquisadores, previamente treinados para administrar a escala estabeleceram um bom rapport com os participantes.

As questões da escala de estratégias de aprendizagem foram gravadas em fita cassete, de forma a se evitar a interferência do pesquisador, bem como facilitar a sua utilização uniforme pelos três pesquisadores da equipe. As instruções e as questões da escala foram ouvidas pelos participantes. As crianças foram orientadas sobre como preencher a escala e caso tivessem dúvidas que levantassem a mão para que as mesmas fossem esclarecidas.

A escala foi aplicada em classes completas, todavia, em pequenos grupos compostos de três a cinco alunos para alunos de $3^{\mathrm{a}}$ e $4^{\mathrm{a}}$ séries e em grupos um pouco maiores para alunos de $5^{\text {a }}$ série em diante, sempre na presença de um aplicador treinado para garantir a objetividade e a homogeneidade na coleta de dados. A aplicação da escala durou em torno de 20 minutos. Cabe mencionar que optou-se por incluir, nesse estudo, somente alunos de $3^{\mathrm{a}}$ série a $8^{\mathrm{a}}$ série, uma vez que a escala era relativamente longa e seria aplicada coletivamente e não individualmente como ocorreu com a entrevista utilizada na coleta de dados do Estudo 1.

\section{Resultados e Discussão}

Para investigar as propriedades psicométricas da escala, em termos de validade e precisão, recorreu-se aos métodos da análise fatorial exploratória, correlação bivariada de $P e$ arson e às estatísticas descritivas. Para verificar se haveria a possibilidade de aplicar a análise fatorial, realizou-se o Teste de Esfericidade de Bartlett, que indicou uma correlação entre os itens $\left(X^{2}[780, N=432]=2549,436 ; p<0,000\right)$. A medida de adequação da amostra foi averiguada pelo índice de Kaiser-Meyer-Olkin (KMO) que foi de 0,758.

A análise fatorial por componentes principais e rotação varimax revelou inicialmente a existência de três fatores com eigenvalues acima de 1,50, capazes de explicar $27 \%$ da variância total. Decidiu-se pela exclusão dos itens que apresentaram saturação em mais de um fator, bem como daqueles com carga fatorial menor que 0,35 . Na Tabela 1 estão apresentados os itens resultantes.

Utilizou-se também do scree test para avaliar a conveniência da manutenção do número de fatores a serem utilizados. Considerando principalmente a coerência teórica dos 
Tabela 1. Distribuição dos itens por fator com a carga fatorial apresentada

\begin{tabular}{|c|c|c|c|}
\hline Itens da escala & F 1 & F 2 & F 3 \\
\hline 37-Você costuma se distrair ou pensar em outra coisa quando está lendo ou fazendo o dever de casa? & 0,679 & & \\
\hline 31-Você costuma ficar pensando em outra coisa quando o professor está dando explicações? & 0,666 & & \\
\hline 38-Você costuma "se esquecer" de fazer o dever de casa? & 0,620 & & \\
\hline 28-Você costuma estudar ou fazer o dever de casa na "última hora? & 0,597 & & \\
\hline 32-Você escuta música ou assiste televisão enquanto estuda ou faz a lição de casa? & 0,586 & & \\
\hline 30-Você costuma desistir quando uma tarefa é difícil ou chata? & 0,507 & & \\
\hline 35-Você costuma comer enquanto estuda ou faz o dever de casa? & 0,451 & & \\
\hline 40-Você se sente cansado quando lê, estuda ou faz o dever de casa? & 0,429 & & \\
\hline 7-Quando você lê um texto, procura escrever com suas palavras o que entendeu da leitura, para poder estudar depois? & & 0,639 & \\
\hline 25-Você costuma ler outros textos e livros sobre o assunto que o professor explicou em aula? & & 0,634 & \\
\hline 5-Você resume os textos que o professor pede para estudar? & & 0,606 & \\
\hline 16-Você costuma fazer um esquema usando as idéias principais do texto? & & 0,561 & \\
\hline 12-Você cria perguntas e respostas sobre o assunto que está estudando? & & 0,538 & \\
\hline 2-Você costuma grifar as partes importantes do texto para aprender melhor? & & 0,363 & \\
\hline 4-Você percebe quando está com dificuldade para aprender determinados assuntos ou matérias. & & & 0,604 \\
\hline 27-Quando você percebe que não entendeu o que leu, você costuma parar e ler novamente? & & & 0,553 \\
\hline 26-Você percebe quando não entende o que está lendo? & & & 0,547 \\
\hline 6-Quando você estuda, você percebe se não está conseguindo aprender? & & & 0,540 \\
\hline 21-Você costuma pedir ajuda ao colega ou a alguém de sua casa, Quando não entende alguma matéria? & & & 0,503 \\
\hline 17-Quando você estuda, você consegue perceber o quanto está aprendendo? & & & 0,433 \\
\hline
\end{tabular}

itens e o gráfico de sedimentação, apresentado na Figura 1, decidiu-se pela manutenção dos três fatores como a melhor configuração para a escala.

Excluídos os itens com saturação inferior a 0,35, o Fator 1 reuniu oito itens $(28,30,31,32,35,37,38$ e 40), cujos conteúdos referem-se à ausência de utilização de estratégias

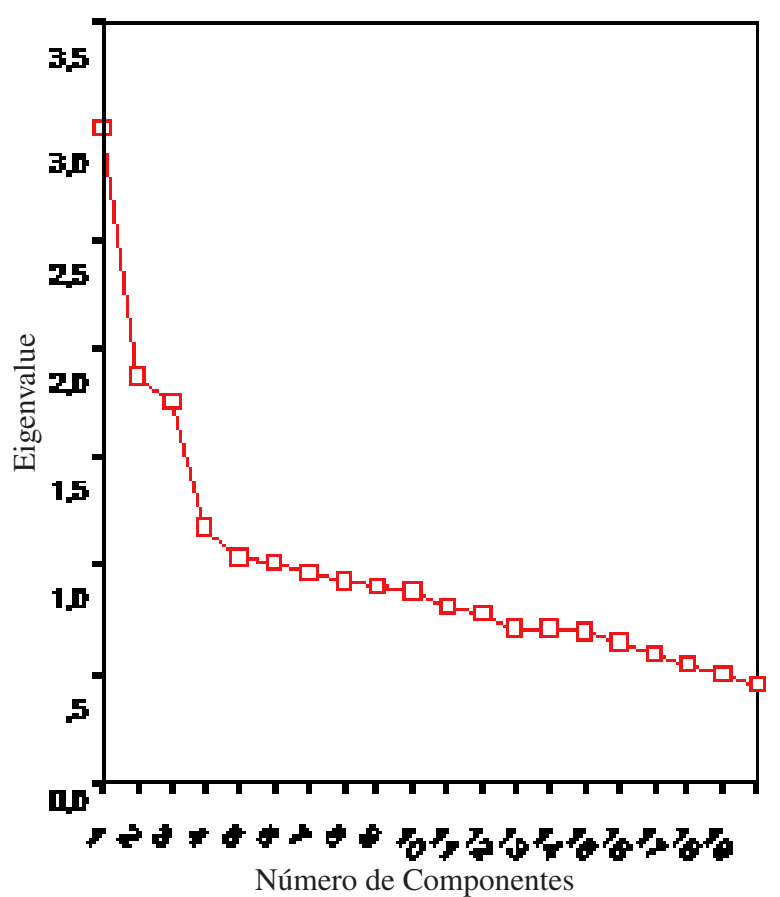

Figura 1. Gráfico de sedimentação com os eingenvalues obtidos de aprendizagem. Interessante destacar que se agruparam nesse fator apenas itens que precisaram ter o valor invertido para a análise, ou seja, elaborados com a intenção de apresentarem comportamentos negativos para a aprendizagem. O Fator 2 englobou seis itens (2, 5, 7, 12, 16 e 25), sendo todos eles relacionados a estratégias cognitivas. $\mathrm{O}$ Fator 3 reuniu os itens 4, 6, 17, 21, 26 e 27 com conteúdos característicos das estratégias metacognitivas, envolvendo basicamente a consciência de situações em que não estão conseguindo alcançar a aprendizagem pretendida.

Com relação à precisão, a escala total alcançou um índice satisfatório, com coeficientes de Cronbach igual a 0,60. $\mathrm{O}$ primeiro fator foi o que alcançou o maior índice $\alpha=0,71$ e os fatores 2 e 3 ficaram respectivamente $\operatorname{com} \alpha=0,59$ e $\alpha=0,51$. Tais índices apontam para a necessidade de que a escala seja submetida a novos estudos para que se possa avaliar melhor o grau de precisão desses itens. É possível que em uma aplicação da escala com a nova configuração e reduzida à metade do número de itens originais e com um maior número de participantes, os índices de precisão obtidos sejam mais altos do que os que agora foram identificados (Prieto, \& Muñiz, 1999).

Os 20 itens finais, ao se agruparem em três fatores, revelam congruência da escala com os construtos teóricos que ela se propõe a medir. Assim, cada item representa a presença ou ausência das principais estratégias de aprendizagem cognitivas (ensaio, elaboração e organização) e metacognitivas existentes na literatura (planejamento, monitoramento e regulação dos processos cognitivos, afetivos e motiva- 


\section{E. Boruchovitch e cols}

cionais). Esses resultados diferem dos dados encontrados por Santos e cols. (2004), em estudo realizado com alunos universitários no qual foram identificados cinco fatores. É possível que o construto estratégias de aprendizagem em crianças seja menos complexo do que em amostras mais avançadas de idade. Futuros estudos devem examinar o impacto que as diferenças desenvolvimento podem apresentar, apontando novas evidências de validade de construto.-

\section{Análise de conteúdo da questão aberta}

Dos 433 estudantes, a maioria $(91,5 \%)$ afirmou não usar nenhuma outra estratégia de aprendizagem. Somente 37 participantes $(8,5 \%)$ responderam à questão aberta. A maioria dos respondentes $(n=32)$ mencionou algumas estratégias que já constavam na escala de estratégia de aprendizagem como, por exemplo, procurar um lugar calmo para estudar, fazer perguntas a respeito da matéria estudada, prestar atenção no que o professor diz, fazer perguntas ao professor diante de dúvidas e entender as figuras para compreender o texto. Atividades como, brincar de escolinha, decorar a matéria, pedir para os pais "tomarem" a lição, ler a matéria e depois fechar o caderno e falar tudo o que entendeu, estudar em grupo, usar o gravador, usar o computador, listar dúvidas, entre outras estratégias, foram citadas por apenas cinco estudantes. Portanto, pode ser constatado que, entre as estratégias mencionadas pelos participantes na questão aberta, houve predomínio daquelas cujo conteúdo era similar aos itens já propostos na escala.

\section{Considerações Finais}

A escala de estratégias de aprendizagem para alunos do ensino fundamental representa uma primeira tentativa de se medir o construto estratégias de aprendizagem por meio de um instrumento nacional. Acredita-se que esta escala possa se constituir num instrumento útil para avaliação, diagnóstico e intervenção em Psicologia Escolar e em Psicopedagogia. Tendo em vista que os índices de consistência interna, obtidos para a escala e subescalas, não terem sido elevados, faz-se necessário um exame mais aprofundado da sua estrutura, de forma a se verificar a sua consonância e validade empírica com os construtos que se propõe a medir, bem como uma aplicação da escala em amostras maiores e mais representativas, em nível nacional.

Importante considerar que pelo fato do instrumento ter como objetivo avaliar o uso de estratégias em estudantes do ensino fundamental, o número de itens inicialmente propostos pode ter sido um elemento que a tenha tornado cansativa e dificultado respostas mais atentas das crianças. Poder-seá, possivelmente, verificar em futuras pesquisas em que a escala seja aplicada com sua formulação final com 20 itens que os participantes respondam com mais facilidade e atenção, visto que seriam poucas frases. Estudos com a versão reduzida da escala em amostras mais jovens como alunos de $1^{\mathrm{a}}$ e $2^{\mathrm{a}}$ série podem também trazer resultados interessantes quanto ao alcance do instrumento, sendo, portanto, recomendados. Novas análises que garantam maior fidedignidade dos itens devem ainda ser empreendidas para que o uso do instrumento possa realmente fornecer informações confiáveis.

Futuros estudos devem ser orientados no sentido de se avaliar os alcances e as limitações do uso da escala para diagnóstico, intervenção e prevenção na área de aprendizagem, no ensino fundamental. Por um lado, espera-se que o uso desta escala possa trazer contribuições, fornecendo informações válidas e confiáveis para que ações pedagógicas, visando à melhoria e à prevenção de problemas relativos ao rendimento acadêmico de alunos do ensino fundamental possam ser implementadas. Por outro lado, sua utilização também poderá contribuir para a implementação de ações preventivas de desenvolvimento da capacidade metacognitiva e da auto-regulação da aprendizagem de estudantes.

Assim, acredita-se que o investimento na construção e no refinamento de um instrumento dessa natureza abre avenidas importantes para o campo da psicologia educacional e das práticas educativas. Representa não só uma tentativa de resposta necessária à carência de instrumentos nacionais de avaliação psicoeducacional, tendo como referencial teórico a Psicologia Cognitiva contemporânea, mas também uma contribuição para a ampliação da discussão e produção de conhecimento nessa área.

\section{Referências}

Bardin, L. (1991). Análise de conteúdo (L. A. Reto \& A. Pinheiro, Trad.) Lisboa: Edições 70. (Trabalho original publicado em 1977)

Berelson, B. (1952). Content analysis in communications research. Glencoe: Free Press.

Boruchovitch, E. (1993). A Psicologia cognitiva e a metacognição: novas perspectivas para o fracasso escolar brasileiro. Tecnologia Educacional, 22(110/111), 22-28.

Boruchovitch, E. (1995). A identificação e o estudo das variáveis associadas ao fracasso escolar brasileiro. Projeto de pesquisa (CNPq- processo No 300162/95-2). Faculdade de Educação, Departamento de Psicologia Educacional, Universidade Estadual de Campinas, Campinas-SP.

Boruchovitch, E. (1999). As estratégias de aprendizagem e o desempenho escolar de crianças brasileiras: considerações para a prática educacional. Psicologia: Reflexão e Crítica, 12(2), 361-376.

Boruchovitch, E. (2001). Algumas estratégias de compreensão em leitura de alunos do ensino fundamental. Psicologia Escolar e Educacional, 5(1), 19-25.

Boruchovitch, E. \& Schall, V. T. (1999). Questionnaires in health education research. Advantages and disadvantages of openended questions. Implications for health research methodology. Ciência e Cultura, 51(1), 12-15.

Boruchovitch, E. \& Santos, A. A. A. (2001). Escala de estratégias de aprendizagem para alunos do ensino fundamental. Manuscrito não publicado, desenvolvido em projeto de intercâmbio interinstitucional -UNICAMP-USF.

Brasil, Lei 9.394, de 20 de dezembro de 1996. Diretrizes e Bases da Educação Nacional, Brasília: 1996. 
Chakur, C. R. S. L (1998). Tarefa escolar: o que aluno registra em seu caderno [Resumo]. Em Sociedade Brasileira de Psicologia (Org.). Resumos de comunicações científicas da XXVIII Reunião Anual de Psicologia (p.159), Ribeirão Preto-SP.

Conselho Nacional de Saúde. Ministério da Saúde (1996). Resolução $n^{\circ}$ 196/96 sobre pesquisa envolvendo seres humanos, Brasília: DF. Publicada em 10 de outubro de 1996.

Costa, E. R., \& Boruchovitch, E. (2004). Compreendendo as relações entre estratégias de aprendizagem e ansiedade de alunos do ensino fundamental de Campinas. Psicologia Reflexão $e$ Crítica, 17(1), 15-24.

Costa, E. R. (2000). As estratégias de aprendizagem e a ansiedade de alunos do ensino fundamental: implicações para a prática educacional. Dissertação de Mestrado, Faculdade de Educação, Universidade Estadual de Campinas, Campinas.

Cruvinel, M. \& Boruchovitch, E. (2004). Sintomas depressivos e estratégias de aprendizagem e rendimento escolar de alunos do ensino fundamental. Psicologia em Estudo, 9(3), 331-338.

Cruvinel, M. (2003). Depressão infantil, rendimento escolar, e estratégias de aprendizagem de alunos do ensino fundamental. Dissertação de Mestrado, Faculdade de Educação, Universidade Estadual de Campinas. Campinas.

Da Silva A. L. \& De Sá, L. (1997). Saber estudar e estudar para saber. Coleção Ciências da Educação. Porto: Porto Editora.

Dembo, M. H. (1994). Applying educational psychology ( $5^{\mathrm{a}} \mathrm{ed}$.). New York: Longman Publishing Group.

Escolano, A. C. M. \& Linhares, M. B. M (1998).Estratégias de busca de informação em situação de resolução de problema em crianças da $1^{\text {a }}$ série do primeiro grau. [Resumo]. Em Sociedade Brasileira de Psicologia (Org.), Resumos de comunicações científicas da XXVIII Reunião Anual de Psicologia (p.160), Ribeirão Preto: SP.

Garner, R. \& Alexander, P. A. (1989). Metacognition: Answered and unanswered questions. Educational Psychologist, 24(2), 143-158.

Garner, R., Hare, V. C., Alexander, P., Haynes, J. \& Winograd, P. (1984). Inducing use of a text lookback strategy among unsucessful readers. American Educational Research Journal, 21, 789-798.

Gombi, R. B. O. (1999). Orientações motivacionais e emprego de estratégias de aprendizagem no estudo da disciplina Sociologia em cursos superiores. Dissertação de Mestrado em Educação, Universidade Estadual de Londrina, Londrina.

Gomes, M. A. A. (2002). Aprendizagem auto-regulada da leitura numa perspectiva de jogos de regras. Dissertação de Mestrado, Faculdade de Educação, Universidade Estadual de Campinas, Campinas.

Gomes, M. A M. \& Boruchovitch, E. (2005). Desempenho no jogo, estratégias de aprendizagem e compreensão na leitura. Psicologia Teoria e Pesquisa, 21(3), 319-326.

Günther, H. \& Lopes Jr., J. (1990). Perguntas abertas versus perguntas fechadas: uma comparação empírica. Psicologia: Teoria e Pesquisa, 6, 203-213.

Hattie, J. Biggs, J. \& Purdie, N. (1996). Effects of learning skills interventions on students: A meta analysis. Review of Educational Research, 66(2), 99-136.

Isaac, S. \& Michael, W.B. (1982). Handbook in research and evaluation. San Diego: Edits I \& M.
Jalles, C. M. C. R.. (1997). O efeito de instruções sobre estratégias metacognitivas de crianças pré-escolares em solução de problema geométrico. Dissertação de Mestrado. Faculdade de Educação, Universidade Estadual de Campinas, Campinas.

Joly, M. C. R. A., Cantalice, L.M. de \& Vendramini, C. M. M. (2004). Evidências de validade de uma escala de estratégias de leitura para universitários. Interação em Psicologia, 8, 261-270.

Joly, M.C.R.A. \& Paula, L.M. de (2005). Avaliação do uso de estratégias de aprendizagem e a compreensão em leitura de universitários. Em M. C. R. A. Joly, A. A. A. Santos \& F. F. Sisto (Orgs.), Questões do cotidiano universitário (pp.33-58). São Paulo: Casa do Psicólogo.

Lopes, M. C. C. (1997). O uso de estratégias cognitivas e metacognitivas no ensino/ aprendizagem da leitura no 1o Grau: uma proposta de intervenção. Dissertação de Mestrado, Instituto de Estudos da Linguagem, Universidade Estadual de Campinas, Campinas.

Machado, O. A. (2005). Evasão de alunos de cursos superiores: fatores motivacionais e de contexto. Dissertação de Mestrado em Educação, Universidade Estadual de Londrina, Londrina.

Molina, O. (1983). Desenvolvimento de habilidades de estudo: uma estratégia ao alcance do professor. Educação e Seleção, $8,45-53$.

Molina, O. (1984). Diferenças no desempenho em leitura com resultado de treinamento em habilidades de estudo. Educação e Seleção, 10, 35-42.

Noronha, A. P. P. (2002). Problemas mais graves e mais freqüentes no uso dos testes psicológicos. Psicologia Reflexão e Crítica, 15 (1), $135-142$.

Noronha, A. P. P., Oliveira, K. L. \& Beraldo, F. N. M. (2003). Instrumentos psicológicos mais conhecidos e utilizados por estudantes e profissionais de Psicologia. Psicologia Escolar e Educacional, 7(1), 47 - 56.

Pintrich, P. R. \& Groot, E. V. (1989). Motivational and SelfRegulated Learning Components of Classroom Academic Performance. Journal of Educational Psychology, 82(1), 33-40.

Pozo, J. J. (1996). Estratégias de Aprendizagem. Em C. Coll, J. Palácios \& A. Marchesi, (Orgs.), Desenvolvimento psicológico e educação: psicologia da educação (pp. 176-197). Porto Alegre:Artes Médicas.

Pressley, M. \& Levin, J. R. (1983). Cognitive strategy research: Psychological Foundations. New York: Springer-Verlag.

Prieto, G. \& Muñiz, J. (1999). Um modelo para evaluar la calidad de los tests utilizados em España. Retirado em 14/02/06 no http://www.cop.es/tests/modelo.htm

Purdie, N. \& Hattie, J. (1996). Cultural differences in the use of strategy for self-regulated learning. American Educational Research Journal, 33(4), 845-871.

Santos, A. A. A. \& Boruchovitch, E. (2001). Escala de estratégias de aprendizagem para alunos universitários. Manuscrito não publicado, desenvolvido em projeto de intercâmbio interinstitucional -UNICAMP-USF.

Santos, A. A. A., Boruchovitch, E., Primi, R, Bueno, J. M. H. \& Zenorini, R. P. C. (2004). Escala de Avaliação de Estratégias de aprendizagem para universitários (EAP-U): Aplicação do modelo de Rasch de Créditos Parciais. Psicologia: Teoria, Investigação e Prática, 9(2), 227-242. 
Schlieper, M. D. M. J. (2001). As estratégias de aprendizagem e as atribuições de causalidade de alunos do ensino fundamental. Dissertação de Mestrado, Universidade Estadual de Campinas, Campinas.

Weinstein, C. E. \& Mayer, R. E. (1985). The teaching of learning strategies. Em M. Wittrock (Org.), Handbook of research on teaching (pp.315-327). New York: Macmillan.

Weinstein, C. E. \& Palmer, D. R. (1987). Learning and Study Strategies Inventory (LASSI) Clearwater: H \& H Publishing Company.

Weinstein, C. E. \& Palmer, D. R. (1990).Learning and Study Strategies Inventory (LASSI) Clearwater: H \& H Publishing Company.

Zimmerman, B. J. \& Martinez-Pons, M. (1986). Development of a structured interview for assessing student use of self-regulated learning strategies. American Educational Research Journal, $23,614-628$.
Zimmerman, B. J. (1986). Becoming a self-regulated learner: Which are the key subprocesses? Contemporary Educational Psychology, 1, 307-313.

Zimmerman, B. J. (1998). Academic studying and the development of personal skill: A self- regulatory perspective. Educational Psychologist, 33(2/3), 73-86.

Recebido em 15.06.2005 Primeira decisão editorial em 13.01.2006 Versão final em 02.06.2006 Aceito em 13.11.2006

\section{MUDANÇA DE ENDEREÇO}

Se você está mudando de endereço, por favor, notifique Psicologia: Teoria e Pesquisa. Ocasionalmente, assinantes e autores comunicam tardiamente mudança de endereço gerando dificuldades na entrega de nossa revista ou de outras correspondências. Informe imediatamente, se possível com antecedência, qualquer mudança de domicílio para:

Universidade de Brasília - Instituto de Psicologia

Revista Psicologia: Teoria e Pesquisa

Secretaria de Divulgação

70910-900 Brasília DF

Mudanças de endereço poderão também ser comunicadas através do correio eletrônico revptp@unb.br ou do telefone e fax 556132746455 . 